\title{
Effect of Anacardium occidentale L. (Cashew Nuts) on Blood Lipids in Indian Subjects
}

\author{
Surendra Kumar Verma ${ }^{1}$, Nita Sahi ${ }^{1}$ and Vartika Jain ${ }^{2 *}$ \\ ${ }^{1}$ Department of Medicine and Department of Biochemistry, Pacific Medical College \\ and Hospitals, Udaipur, Rajasthan, India \\ ${ }^{2}$ Department of Botany, Government Meera Girls College, Udaipur, Rajasthan, India \\ *Corresponding Author: Vartika Jain, Department of Botany, Government Meera \\ Girls College, Udaipur, Rajasthan, India.
}

Received: September 12, 2021

Published: October 26, 2021

(C) All rights are reserved by Surendra Kumar Verma., et al.

\begin{abstract}
Nuts are part of Indian diet as luxurious and nutritious food items and Anacardium occidentale L. (Cashew nuts) is one such important nut consumed raw or in various sweet dishes Thirty, male healthy individuals was selected for the present study. After taking initial fasting blood samples, they were administered $10 \mathrm{~g}$ of Cashew nuts daily for four weeks and again the blood samples were collected. The Cashew nuts administration was discontinued and the blood samples were again collected after four weeks. There was decrease in total cholesterol (3.2\%), triglycerides (7.9\%), LDL-C (6.58\%), VLDL-C (7.8\%) but the reduction was not statistically significant ( $\mathrm{p}=\mathrm{NS}$ ). HDL-C was also not altered significantly. On further analysis of the data based on the levels of total cholesterol (less than or more than $200 \mathrm{mg} / \mathrm{dl}$ ), the effect of Cashew nuts was more pronounced and statistically significant (p < 0.05) on individuals with higher lipids. This is probably first report highlighting the significant hypolipidemic effects of Cashew nut in a small dose. However, further studies on larger number of individuals in different doses are warranted.
\end{abstract}

Keywords: Cholesterol; LDL-C; Non HDL-C; Atherogenic Lipids; Nuts

\section{Introduction}

Dyslipidemia and low antioxidant status are some of the major culprits behind cardiovascular diseases (CVD) besides, high blood pressure, high blood glucose and obesity which are other important risk factors. Plants have always been employed in traditional medicinal systems for treatment of many infectious as well as non-infectious ailments. Scientific validation studies on many of the plant species have proven their efficacy against coronary heart disease and CVD [1-7].

Nuts or dried fruits obtained from plants such as Prunus amygdalus, Juglans regia, Pistacia vera, Anacardium occidentale etc. are occasional small luxuries which are enjoyed by people as whole and also incorporated in different food items such as sweets, chocolates, shakes etc. [8]. Interestingly, many of the nuts due to their nutritional and phytochemical composition have demonstrated lipid lowering and antioxidant potential in animal and human studies. For example, Almonds have shown to reduce total cholesterol (TC), low density lipoprotein-cholesterol (LDL-C) and improve levels of high density lipoprotein-cholesterol (HDL-C) in persons having dyslipidemia [9]. Similarly, Walnuts have also shown hypotensive, hypolipidemic, anti-diabetic, antioxidant, and liver protective activities [10]. Beneficial effect of nuts consumption have also been observed on TC, LDL-C, endothelial function in various cardiometabolic diseases [11]. Hong et al. [12] have demonstrated that eight weeks consumption of mixed nuts having Almonds, Peanuts, Cashews, Brazil nuts, Macadamia nuts and single nut Pistachio sig- 
nificantly reduce $\mathrm{TC}$, LDL-C, triglycerides, c-reactive protein and oxidative stress in animals.

There are few studies on Cashew nuts regarding their controversial role on lipid profile and creates a question mark on efficacy of Cashew nuts on reducing various lipid parameters [13-16]. In view of this, effect of consumption of Cashew nuts on lipid profile was studied in healthy volunteers.

\section{Materials and Methods}

\section{Study protocol}

After approval from institutional ethical committee, the study was conducted on 30 healthy individuals between the ages of 30 to 60 years. All the volunteers were non-obese $(\mathrm{BMI}<24)$, nondiabetic, non-hypertensive, non-alcoholic and non-smoker. They were not taking tobacco or any drugs affecting blood lipids and weight reducing drugs. Study was conducted in accordance with the guidelines of the Declaration of Helsinki and Tokyo, 2004. The study subjects were selected from the medical Out Patient Department of Medicine, Pacific Medical College and Hospitals, Udaipur. All the study subjects were not allowed to alter their dietary and exercise schedule and take any drug without consultation during the study period.

\section{Administration of Cashew nuts}

Good quality raw processed Cashew nuts Anacardium occidentale L. (Family - Anacardiaceae) were purchased from the local market. These were identified and authenticated at Department of Botany, Govt. Meera Girls College, Udaipur. After obtaining written consent, Cashew nuts were administered to all the study subjects in a dose of $10 \mathrm{~g} /$ day for four weeks regularly. It was advised to take these in the morning with the instruction to crush and grind properly in the mouth. During the entire study period, the participants were instructed not to take any other nuts.

\section{Blood chemistry}

Blood samples were collected in a fasting state, initially and at the end of $4^{\text {th }}$ and $8^{\text {th }}$ weeks for the analysis of lipid profile including serum Total cholesterol (TC), Triglycerides (TG) and High density lipoprotein cholesterol (HDL-C) by the standard methods [17-19]. Very low density lipoprotein cholesterol (VLDL-C) and Low density lipoprotein cholesterol (LDL-C) were calculated by Friedwald formula [20] as follows:
VLDL-C = Triglycerides $/ 5$

LDL-C = Total cholesterol - (HDL-C + VLDL-C)

Non HDL-C = Total cholesterol - HDL-C

\section{Statistical analysis}

All the data were expressed as mean \pm standard error (SE). Results were statistically analyzed with student's t-test and a 'p' value less than 0.05 was considered as significant difference in analysis.

\section{Results and Discussion}

Administration of $10 \mathrm{~g}$ Cashew nut to healthy volunteers for 4 weeks led to some alterations in lipid parameters. There was decrease in Total Cholesterol (3.2\%), Triglycerides (7.9\%), LDL-C (6.58\%), VLDL-C (7.8\%) and non HDL-C (4.5\%) with minimal changes in HDL-C, TC/HDL-C and LDL-C/HDL-C ratio. All the changes were statistically not significant $(p=N S)$. These alterations in lipid fractions, although statistically not significant, yet are important in view that when the levels were repeated 4 weeks after discontinuation of Cashew nuts, all parameters were significantly $(p<0.05)$ increased. These observations point toward beneficial effect of Cashew nut administration in disguise (Table 1, Figure 1).

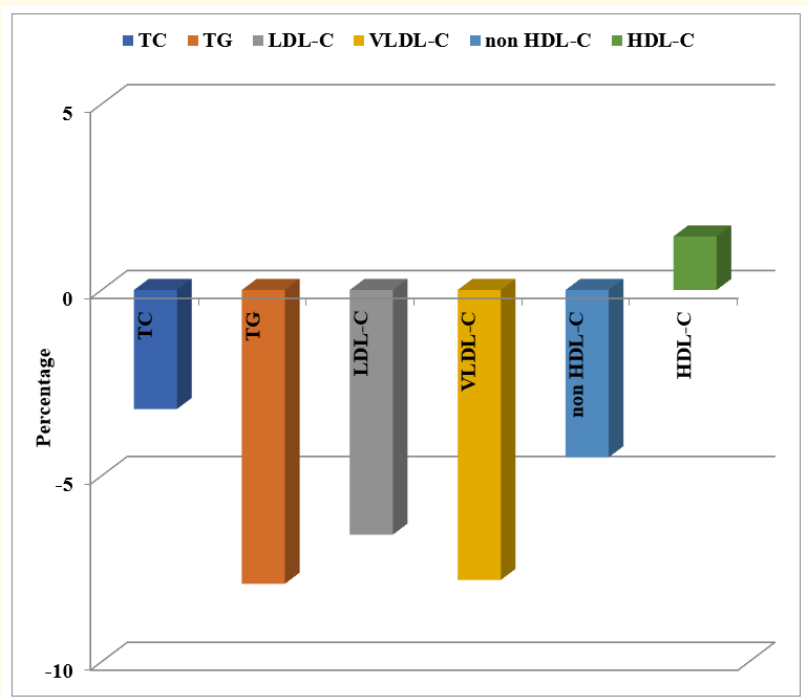

Figure 1: Percent change in various lipid fractions after 4 weeks of $10 \mathrm{~g}$ Cashew nut consumption $(\mathrm{n}=30)$. 


\begin{tabular}{|l|c|c|c|c|c|}
\hline Parameter & Initial & After 4 weeks & p value* & $\begin{array}{c}\text { Four weeks after } \\
\text { stopping of nut }\end{array}$ & p value\# \\
\hline $\begin{array}{l}\text { Total cholesterol (TC) } \\
\text { (mg/dl) }\end{array}$ & $191.27 \pm 8.31$ & $185.1 \pm 8.6$ & NS & $197.1 \pm 8.37$ & $<0.05$ \\
\hline Triglycerides (mg/dl) & $113.67 \pm 11.75$ & $104.6 \pm 9.03$ & NS & $140.56 \pm 16.92$ & $<0.05$ \\
\hline HDL-C (mg/dl) & $41.4 \pm 1.95$ & $42.0 \pm 1.88$ & NS & $36.96 \pm 1.09$ & NS \\
\hline LDL-C (mg/dl) & $131.67 \pm 7.09$ & $123.00 \pm 9.69$ & NS & $137.62 \pm 8.54$ & $<0.05$ \\
\hline VLDL-C (mg/dl) & $18.19 \pm 1.88$ & $16.76 \pm 1.41$ & NS & $22.42 \pm 2.70$ & $<0.05$ \\
\hline Non HDL-C (mg/dl) & $149.87 \pm 7.5$ & $143.1 \pm 7.8$ & NS & $160.2 \pm 7.1$ & $<0.05$ \\
\hline TC/HDL-C ratio & $4.75 \pm 0.29$ & $4.51 \pm 0.28$ & NS & $5.43 \pm 0.30$ & $<0.05$ \\
\hline LDL-C/HDL-C ratio & $3.29 \pm 0.25$ & $3.02 \pm 0.29$ & NS & $3.81 \pm 0.28$ & $<0.05$ \\
\hline
\end{tabular}

Table 1: Effect of Cashew nut (10 g) on various lipid fractions in healthy volunteers $(n=30)$.

Values are expressed as Mean \pm SE.

*p Value - As compared to initial.

\#p Value - As compared to after 4 weeks of supplementation of Cashews.

HDL-C: High density lipoprotein cholesterol; LDL-C: Low density lipoprotein cholesterol; VLDL-C: Very low density lipoprotein cholesterol.

Further analysis of the data show some interesting results, not yet reported. When these observations were break up into two groups one having TC more than $200 \mathrm{mg} / \mathrm{dl}$ and other with TC less than $200 \mathrm{mg} / \mathrm{dl}$; the results were remarkably different. In group with TC more than $200 \mathrm{mg} / \mathrm{dl}$; reduction in TC (13.48\%),
TG (31.96\%), LDL-C (14.25\%), VLDL-C (28.76\%), and non HDL-C (16.73\%) with minimal alterations in HDL-C, TC/HDL-C and LDL$\mathrm{C} / \mathrm{HDL}-\mathrm{C}$ ratio was observed. All the changes were statistically significant $(\mathrm{p}<0.05)$. The other group with TC less than $200 \mathrm{mg} /$ $\mathrm{dl}$ showed the insignificant alterations in all parameters (Table 2, Figure 2 and 3).

\begin{tabular}{|l|c|c|c|c|c|c|}
\hline \multirow{2}{*}{ Parameters } & \multicolumn{3}{|c|}{ Individuals with TC<200 mg/dl } & \multicolumn{2}{c|}{ Individuals with TC>200 mg/dl } \\
& \multicolumn{2}{|c|}{ (n= 20) } & & \multicolumn{2}{c|}{ (n) } \\
\cline { 2 - 7 } & Initial & After 4 weeks & p value & Initial & After 4 weeks & p value \\
\hline $\begin{array}{l}\text { Total cholesterol } \\
\text { (mg/dl) }\end{array}$ & $172.3 \pm 8.91$ & $174.7 \pm 6.42$ & NS & $229.2 \pm 6.38$ & $198.3 \pm 5.86$ & $=0.001$ \\
\hline Triglycerides (mg/dl) & $97.7 \pm 6.25$ & $103.65 \pm 7.55$ & NS & $150.8 \pm 15.48$ & $102.6 \pm 10.09$ & $<0.05$ \\
\hline HDL-C (mg/dl) & $39.85 \pm 1.37$ & $42.55 \pm 1.58$ & NS & $43.1 \pm 2.8$ & $44.5 \pm 2.67$ & NS \\
\hline LDL-C (mg/dl) & $116.82 \pm 4.09$ & $119.59 \pm 8.22$ & NS & $161.38 \pm 5.31$ & $138.37 \pm 5.75$ & $<0.05$ \\
\hline VLDL-C (mg/dl) & $16.63 \pm 0.78$ & $16.65 \pm 1.18$ & NS & $24.09 \pm 2.48$ & $17.16 \pm 1.87$ & $<0.05$ \\
\hline Non HDL-C (mg/dl) & $133.15 \pm 4.41$ & $132.47 \pm 6.87$ & NS & $187.88 \pm 6.73$ & $156.44 \pm 7.17$ & $<0.05$ \\
\hline TC/HDL-C ratio & $4.45 \pm 0.21$ & $4.37 \pm 0.25$ & NS & $5.33 \pm 0.34$ & $4.78 \pm 0.27$ & NS \\
\hline LDL-C/HDL-C ratio & $3.05 \pm 0.20$ & $2.98 \pm 0.26$ & NS & $3.77 \pm 0.28$ & $3.09 \pm 0.32$ & NS \\
\hline
\end{tabular}

Table 2: Effect of Cashew nut (10 g) on various lipid fractions in healthy volunteers after break up in low and high TC groups.

Values are expressed as Mean $\pm \mathrm{SE}$.

$\mathrm{p}$ Value - As compared to initial.

HDL-C: High density lipoprotein cholesterol; LDL-C: Low density lipoprotein cholesterol; VLDL-C: Very low density lipoprotein cholesterol. 


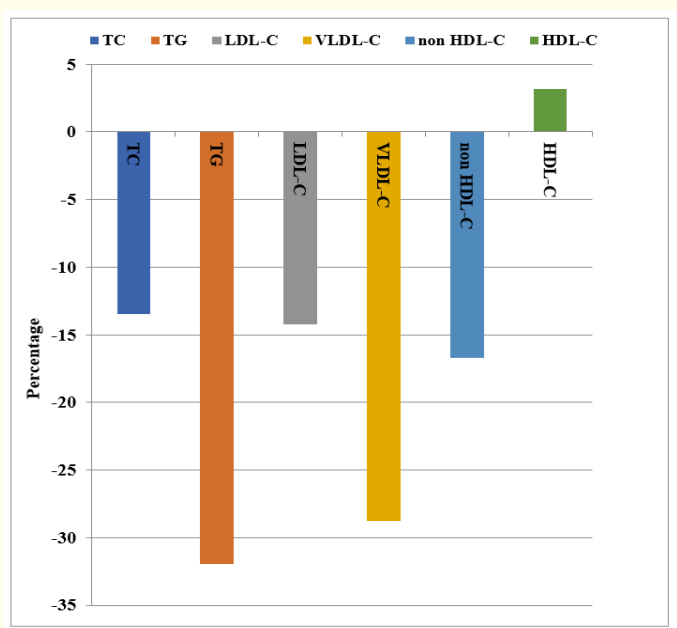

Figure 2: Percent change in various lipid fractions after 4 weeks of $10 \mathrm{~g}$ Cashew nut consumption in individuals with $\mathrm{TC}>200$ $\mathrm{mg} / \mathrm{dl}(\mathrm{n}=10)$.

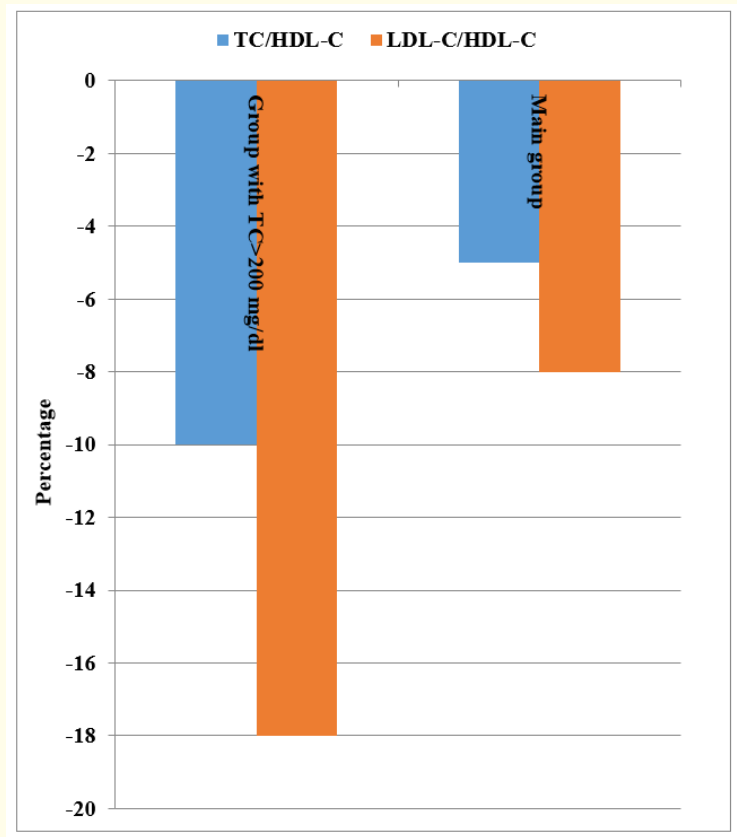

Figure 3: Percent change in TC/HDL-C and LDL-C/HDL-C ratios after 4 weeks of $10 \mathrm{~g}$ Cashew nut consumption in subsets of individuals with $\mathrm{TC}>200 \mathrm{mg} / \mathrm{dl}(\mathrm{n}=10)$ and main study group $(n=30)$.
All the observations point toward the lipid favorable effect of Cashew nuts in healthy individuals. It does not significantly altered the lipid parameters in individuals with normal cholesterol but significantly reduces atherogenic lipids in individuals with high cholesterol; thereby maintaining a normal lipid profile.

Nuts have been approved by US Food and Drugs Administration as a qualified health preserving and for reduction of cardiovascular disease. Unfortunately, Cashews are excluded from that claims probably because of their saturated fat contents, which are palmitic acid (10.3\%) and stearic acid (8.8\%). However, Cashew nuts have abundant monounsaturated fatty acids (oleic acid - 61.8\%) and polyunsaturated fatty acids (linoleic acid - 17.8\%) which are associated with reduced cardiovascular disease risk. Although Cashew nuts are rich in saturated fatty acids but combination of both MUFA and PUFA exceeds the total amount of saturated fatty acids and therefore, may prevent against bad effects of saturated fatty acids. Besides, Cashew nuts also contain polyphenols, tocopherols and Beta-sitosterols which may be instrumental in lowering the lipid fractions in hyperlipidemic individuals (Table 3) [21,22].

\begin{tabular}{|l|c|}
\hline Content & Value \\
\hline Moisture & $4.57 \pm 0.22 \%$ \\
\hline Protein (g/100g) & $18.78 \pm 0.58$ \\
\hline Total oil & $6.4 \pm 1.2 \%$ \\
\hline Total carbohydrates & $29.8 \%$ \\
\hline Palmitic acid & $10.3 \%$ \\
\hline Stearic acid & $8.8 \%$ \\
\hline Oleic acid & $61.8 \%$ \\
\hline Linoleic acid & $17.3 \%$ \\
\hline Total Saturated Fatty Acids (mg/100g) & $7816 \pm 148$ \\
\hline $\begin{array}{l}\text { Total Mono Unsaturated Fatty Acids (MUFA) } \\
\text { (mg/100g) }\end{array}$ & $27907 \pm 309$ \\
\hline $\begin{array}{l}\text { Total Poly Unsaturated Fatty Acids (PUFA) } \\
\text { (mg/100g) }\end{array}$ & $7482 \pm 203$ \\
\hline Total polyphenols (mg/100g) & $32.86 \pm 1.76$ \\
\hline Campesterol (mg/100g) & $3.64 \pm 0.07$ \\
\hline Stigmasterol (mg/100g) & $0.89 \pm 0.04$ \\
\hline$\beta$-Sitosterol (mg/100g) & $110 \pm 2.5$ \\
\hline Tocopherols alpha (mg/100g) & $1.05 \pm 0.05$ \\
\hline Tocopherols beta (mg/100g) & $0.05 \pm 0.01$ \\
\hline Tocopherols gamma (mg/100g) & $5.26 \pm 0.05$ \\
\hline$\alpha$-Tocopherol Equivalent (mg/100g) & $1.05 \pm 0.06$ \\
\hline
\end{tabular}

Table 3: Chemical composition of Cashew nuts [21,22]. 
Several studies have been conducted to observe the effect of Cashew nuts on various fractions of blood lipids in human and animal studies with conflicting results. Consumption of Cashew nuts in the dose of $42 \mathrm{~g}$ daily in 1.5 servings in adult subjects for 4 weeks did not alter the lipid levels to any significant extent [14]. In a parallel, randomized controlled study on 30 adult Asian Indian males with type 2 diabetes mellitus where control group was assigned a standard diabetic diet and the intervention group received similar diet plus $30 \mathrm{~g}$ Cashew nuts daily for 12 weeks. There was greater increase in plasma HDL-C compared with control ( $p=0.01)$. There was no effect on blood sugar and other lipid parameters [23].

Systemic review and Meta-analyses of six randomized clinical trials comprising 531 subjects for systemic review and three studies were included in the meta-analyses model from inception until June 2019. The results of the analyses demonstrated no significant changes in TC, TG, LDL-C and HDL-C [15]. In a randomized, crossover, controlled trial on 51 men and women with a median LDL-C concentration of $159 \mathrm{mg} / \mathrm{dl}$ showed that supplementation of Cashew nuts (28-64 g/day) to the typical American diets decreases TC \& LDL-C more than control group ( $\mathrm{p}<0.05)$. However, TG and HDL-C did not show any significant alterations [13].

Study by Mah., et al. [13] throws some light on our observations which were analyzed in volunteers with base line TC>200 mg/dl. Their mean LDL-C $(161.38 \pm 5.31)$ was also consistent with the LDL-C levels of study group. In spite of a very high dose of Cashew nuts (28-64 g/day), their reduction in TC and LDL-C was 3.9\% and $4.8 \%$ respectively. On the other hand, the present study has clearly shown a reduction in TC (13.48\%) and LDL-C (14.25\%) even with the $10 \mathrm{~g}$ of Cashew nuts. On analysis of all the human studies of Cashew nuts, the variability of the effect on lipid fraction is probably due to the dose supplemented $42 \mathrm{~g}$ [14], $30 \mathrm{~g}$ [23], 28-64 g [13] and the duration of the study varying for 4 weeks to 12 weeks.

The present study did not aim to unveil the hypolipidemic mechanism of Cashew nut and moreover, there is no definite study regarding the mechanisms involved in cholesterol reducing effect of Cashew nuts. However, an experimental study conducted in cell culture carried out by pre-treating hepatoma cells (Hep G2) with different concentrations of aqueous extracts of leaves of Anacardium occidentale leaves did not significantly increase the concentration of LDL receptors but significantly increased the concen- tration of Farnesyl diphosphate farnesyl transferase (FDFT1), Apolipoprotein A1 (APO A1), Lecithin-cholesterol acyltransferase (LCAT), Scavenger receptor-B 1 (SR-B1) etc. [24]. These findings suggest that leaves of $A$. occidentale may involve in reverse cholesterol transport process to reduce cholesterol metabolism in Hep G2 cells. Role of other phytochemicals present in Cashew nuts which are known for their hypolipidemic effect should also be evaluated in future studies.

The conclusion emerging from all these studies is that Cashew nut administration in normolipidemic individuals does not alter the lipid fractions while in hyperlipidemic group; it reduces the elevated lipid levels significantly. Therefore, it can be administered to both subsets of individuals one with lipid neutral effects and other with hypolipidemic action.

\section{Conclusion}

Cashew nut supplementation in a dose of $10 \mathrm{~g}$ daily does not alter the lipid parameters in individuals with normal cholesterol levels. But, it significantly reduces raised lipid fractions and ratios in hyperlipidemic individuals. The results are consistently significant in hyperlipidemic subsets of study subjects. However, in the present study, the number of participants is small and therefore, further study on larger number of individuals with higher total cholesterol and LDL-cholesterol is warranted.

\section{Bibliography}

1. Fraser GE., et al. "A possible protective effect of nut consumption on risk of coronary heart disease. The Adventist health study". Archives of Internal Medicine 152 (1992): 1416.

2. Bordia A., et al. "Effect of garlic (Allium sativum) on blood lipids, blood sugar, fibrinogen and fibrinolytic activity in patients with coronary artery disease". Prostaglandins Leukotrienes Essential Fatty Acids 58 (1998): 257-263.

3. Jain V., et al. "Effect of Bombax ceiba root on some cardiovascular parameters in patients with ischemic heart disease". Asian Journal of Biological Sciences 5 (2012): 351-357.

4. Verma SK., et al. "Blood pressure lowering, fibrinolysis enhancing and antioxidant activities of Cardamom (Elettaria cardamomum)". Indian Journal of Biochemistry and Biophysics 46 (2009): 503-506. 
5. Verma SK., et al. "Effect of Greater Cardamom (Amomum subulatum Roxb.) on blood lipids, fibrinolysis, and total antioxidant status in patients with ischemic heart disease". Asian Pacific Journal of Tropical Disease 2.2 (2012): S739-743.

6. Verma SK., et al. "Effect of Pueraria tuberosa DC. (Indian Kudzu) on blood pressure, fibrinolysis and oxidative stress in patients with stage 1 Hypertension". Pakistan Journal of Biological Sciences 15.15 (2012): 742-747.

7. Jain V and Jain SK. Compendium of Indian Folk Medicine and Ethnobotany (1991-2015), Deep Publications, New Delhi, India (2016).

8. Jain V. "Sweets as traditional medicine in winter season: An ethnobotanical study in Udaipur city, India". Ethnobotany Research and Applications (Online) 20 (2020): 1-17.

9. Kalita S., et al. "Almonds and cardiovascular health: A review". Nutrients 10 (2018): 468.

10. Delaviz H., et al. "A review study on phytochemistry and pharmacology applications of Juglans regia plant". Pharmacognosy Reviews 11 (2017): 145-152.

11. Kim Y., et al. "Nuts and cardio-metabolic disease: A review of meta-analyses". Nutrients 10 (2018): 1935.

12. Hong MY., et al. "Anti-inflammatory, antioxidant, and hypolipidemic effects of mixed nuts in atherogenic diet-fed rats". Molecules 23 (2018): 3126.

13. Mah E., et al. "Cashew consumption reduces total and LDL cholesterol: a randomized, crossover, controlled-feeding trial". The American Journal of Clinical Nutrition 105.5 (2017): 10701078.

14. Baer DJ and Novotny JA. "Consumption of cashew nuts does not influence blood lipids or other markers of cardiovascular disease in humans: a randomized controlled trial". The American Journal of Clinical Nutrition 109.2 (2019): 269-275.

15. Morvaridzadeh M., et al. "Effect of Cashew Nut on Lipid Profile: A Systematic Review and Meta-Analysis. Auswirkungen von Cashewnüssen auf das Lipidprofil: Eine systematische Übersicht und Metaanalyse". Complementary Medicine Research 27 (5) (2020): 348-356.
16. Cordaro M., et al. "Cashew (Anacardium occidentale L.) Nuts counteract oxidative stress and inflammation in an acute experimental model of carrageenan-induced paw edema". Antioxidants 9.8 (2020): 660 .

17. Allain CC., et al. "Enzymatic determination of total serum cholesterol". Clinical Chemistry 20 (1974): 470-475.

18. Fossati P and Lorenzo P. "Serum triglycerides determined colorimetrically with an enzyme that produces hydrogen peroxide". Clinical Chemistry 28 (1982): 2077-2079.

19. Izzo C., et al. "Improved method for determination of high density lipoprotein cholesterol I. Isolation of high density lipoproteins by use of polyethylene glycol 6000". Clinical Chemistry 27 (1981): 371-374.

20. Friedwald WT., et al. "Estimation of the concentration of lowdensity lipoprotein cholesterol in plasma, without use of the preparative ultracentrifuge". Clinical Chemistry 18 (1972): 499502.

21. Longvah T., et al. Indian Food Composition Tables. National Institute of Nutrition Indian Council of Medical Research Department of Health Research Ministry of Health and Family Welfare, Government of India Jamai Osmania (PO), Hyderabad, Telangana, India (2017).

22. Griffin LE and Dean LL. "Nutrient composition of raw, dryroasted, and skin-on cashews". Journal of Food Research 6.6 (2017): 13-28.

23. Mohan V., et al. "Cashew Nut Consumption Increases HDL Cholesterol and Reduces Systolic Blood Pressure in Asian Indians with Type 2 Diabetes: A 12-Week Randomized Controlled Trial". Journal of Nutrition 148.1 (2018): 63-69.

24. Hasan MKN., et al. "Anticholesterol activity of Anacardium occidentale Linn. Does it involve in reverse cholesterol transport?" SAINS Malaysiana 44.10 (2015): 1501-1510.

\section{Volume 5 Issue 11 November 2021 \\ (C) All rights are reserved by Surendra Kumar Verma.,} et al. 